\title{
Distribution of some ore metals around the Mau Due stibnite deposits, northernmost Vietnam
}

\author{
Shunso Ishihara ${ }^{1, *}$ and Pham Tich Xuan ${ }^{2}$
}

Shunso Ishihara and Pham Tich Xuan (2013) Distribution of some ore metals around the Mau Due stibnite deposits, northernmost Vietnam. Bull. Geol. Surv. Japan, vol. 64 (1/2), p. 51-57, 4 figures, 3 tables.

\begin{abstract}
Soil and stream sediments were studied geochemically around the Mau Due ore deposits of the northernmost Vietnam. The ore deposits are fracture-filling type occurring in Devonian sediments composed mainly of impure calcareous sediments. The chemical elements brought up by the mineralizations are mainly $\mathrm{S}$ and $\mathrm{Sb}$, and small amount of As and base metal. The analyzed surface samples were collected in the wet season (May to September) and the dry season (October to April).

$\mathrm{Sb}$ contents of surface materials are sporadically high in the soil samples, and have smaller variation in the stream sediments than in the soil samples. Sb contents of the soil of the wet season, $932 \mathrm{ppm} \mathrm{Sb}$ in the average, are higher than the average of $342 \mathrm{ppm} \mathrm{Sb}$ of the dry season. The Sb contents are much higher in the stream sediments, averaged as 2,536 ppm in the wet season and 2,504 ppm in the dry season.

Generally speaking, As contents of the soil are positively correlated with Sb contents, but the amounts are much lower than the $\mathrm{Sb}$ contents, averaged as 29 and $26 \mathrm{ppm}$ in soil of the wet and dry seasons, respectively, but higher as 68 and $67 \mathrm{ppm}$ in the stream sediments. These abundance data of Sb and As are best explained by both elements are present finest grained sulfides in the soil and river sediments. The Devonian host rocks may be originally high in the Sb contents, as compared with the Japanese eugeosynclinal sediments.
\end{abstract}

Keywords: Mau Due mine, stibnite, heavy metals, pollution, Vietnam

\section{Introduction}

Antimony is concentrated in many hydrothermal ore deposits of simple stibnite type and complex sulfidesulphosalts type, and its production is heavily dependent upon the stibnite type in sedimentary terrain in the Xikuangshan area, Hunan province of China, which produces $83-90 \%$ of the world production in the past five years (Ishihara and Ohno, 2011). The element is considered as a good geochemical marker for antimony ore deposits, particularly of the sulfide-sulphosalt type (Boyle and Jonasson, 1984).

Soils and stream sediments around given antimony ore deposit have been polluted by the ore metals, especially of antimony and arsenic (Flynn et al., 2003; Murciego et al., 2007).

In one case of mesothermal antimony deposits, the stibnite can contain up to 5,000 ppm As. Together with common occurrence of arsenopyrite and arsenian pyrite, dissolution of these minerals causes strong contamination of antimony and arsenic into the soil, stream sedi- ments and plants of the antimony mining area (Ashley et al., 2003; Murciego et al., 2007).

In the largest mineralized area of the Hunan province, Wang et al. (2010) examined 10 heavy metals (Sb, As, $\mathrm{Cd}, \mathrm{Cr}, \mathrm{Cu}, \mathrm{Hg}, \mathrm{Mn}, \mathrm{Ni}, \mathrm{Pb}$ and $\mathrm{Zn}$ ) of the surrounding agriculture soils in the mining region, and found that the strongest contamination on $\mathrm{Sb}$ (236 times), $\mathrm{Cd}$ (52 times), $\mathrm{Hg}$ (14 times), and As (3.1 times), Zn (2.9 times) and $\mathrm{Pb}$ (2.5 times), in which cadmium concentration is rather unusual.

In this paper, reconnaissance geochemical work has been done around the Mau Due stibnite mine area. This mine is located at a part of mountainous region, 300 to $1,000 \mathrm{~m}$ above sea level, in the northernmost part of Vietnam (N23 $04^{\prime} 24^{\prime \prime}, \mathrm{E} 105^{\circ} 15^{\prime} 10^{\prime \prime}$, Fig. 1), and are very close to the In-bearing $\mathrm{Sn}-\mathrm{Pb}-\mathrm{Zn}$ deposits of $\mathrm{Du}$ Long mine, China (Ishihara et al., 2011). The Mae Due mine area is composed of two distinct seasons; wet and hot summer, and dry and cool winter. Average rainfall is 1200 to $1800 \mathrm{~mm}$ in a year, in which majority (73-81\%) falls in the rainy season of May to September.

\footnotetext{
${ }^{1}$ AIST, Geological Survey of Japan

${ }^{2}$ Institute of Geological Sciences, Vietnamese Academy of Science and Technology, Hanoi, Vietnam.

*Corresponding author: S. ISHIHARA, Central 7, 1-1-1 Higashi, Tsukuba, Ibaraki 305-8567, Japan. Email: s-ishihara@aist.go.jp
} 


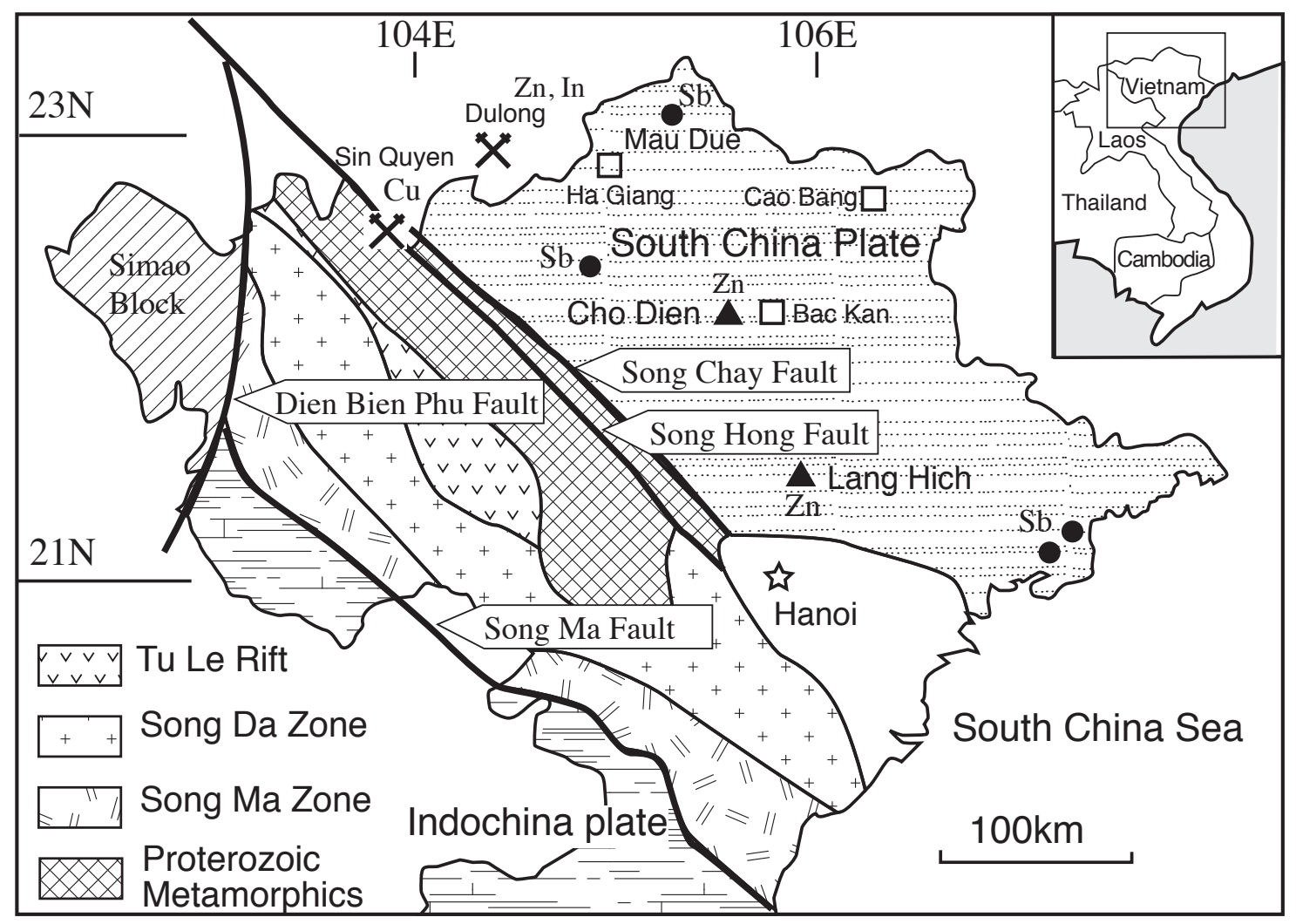

Fig. 1 Index map for the Mau Due mine occurring in the sedimentary terrain of the South China plate.

\section{Geological background}

Northeastern side of the Red River Fault in the northern Vietnam is essentially Paleozoic-Mesozoic sedimentary terrain intruded by Mesozoic granitoids. Small fracture filling-type antimony deposits are known at Lang Vai mine of the central part (500 tons Sb metal, 1986-1992), and Khe Chim, Duong Huy, and Dong Mo localities. In the northernmost part of Ha Giang province, an open pit mining was initiated in 1993 and is called Mau Due mine. A refinery was set up in 2002, and the mine produces annually 100 tons Sb metal since then.

This mine area is underlain by the Devonian $\mathrm{Na}$ Quan Formation (D1-2nq) of about 500 m thick, consisting of limestone alternated with carbonaceous shale and black shales (Tran Xuyen, 2001). The limestone is usually dolomitized. Other Devonian strata of the Toc Tat Formation (D3tt) comprise fine-layered silicic limestone alternated with silicic shale, shale, and carbonaceous shale. The thickness is about $300 \mathrm{~m}$.

The Song Hien Formation (T1sh) is most widely distributed in the mine area. It is composed of sandstone, black shale and sericitic shale. The thickness is about $300 \mathrm{~m}$. Quaternary sediments (Q) are distributed along the Nam Tam river, and are composed of pebbles, sand and clay.

\section{Mineralization at Mae Due mine}

Devonian sediments of the Na Quan Formation (D12nq) which have been folded with NNE-SSW axes and cut by the same NNE-SSW faults, host the stibnite deposits. The stibnite mineralizations are seen along the NNE-SSW structures, and are divided into three orebodies as I, II and III from the west to east.

The orebody I is $350 \mathrm{~m}$ long with the width of $3.7 \mathrm{~m}$ and dip of $70^{\circ} \mathrm{W}$, and average grade of $10.3 \% \mathrm{Sb}$. The orebody II is $410 \mathrm{~m}$ long, $10.1 \mathrm{~m}$ wide dipping $40-70^{\circ}$ west. The average grade is $11.2 \% \mathrm{Sb}$. The orebody III is $200 \mathrm{~m}$ long, $4.1 \mathrm{~m}$ wide, dipping $70^{\circ}$ east. The average grade is $13.6 \% \mathrm{Sb}$. These orebodies are mostly composed of quartz (20-70\%) and small amounts of calcite. The ore minerals are stibnite, and very small amounts of pyrite, arsenopyrite, sphalerite and berthierite. At the top of the orebodies, such secondary minerals as valetinite and lewisite may occur together with limonite.

Some ore components are analyzed for total carbon and sulfur by infrared method, and Fe, Sb, As, Se, $\mathrm{Mo}, \mathrm{Cu}, \mathrm{Pb}, \mathrm{Zn}, \mathrm{Cd}, \mathrm{Bi}, \mathrm{Mn}, \mathrm{Co}$, and $\mathrm{Ni}$ by ICP-MS methods on the ores and wastes of the Mau Due mine, and the results are listed in Table 1. The antimony ores only dominant in $\mathrm{Sb}, \mathrm{S}$, and $\mathrm{Se}$, while other ore elements such $\mathrm{As}, \mathrm{Cu}, \mathrm{Pb}, \mathrm{Zn}, \mathrm{Ni}, \mathrm{Co}$, and $\mathrm{Mo}$ are rich in the wastes, implying that these elements are marginal 
Table 1 Selected ore components of the ores and wastes of the Mau Due mine.

\begin{tabular}{|c|c|c|c|c|c|c|c|c|c|c|c|c|c|c|c|c|c|}
\hline No. & Sample no. \& samples & $\mathrm{Al}$ & $\mathrm{Fe}$ & Total S & Total C & $\mathrm{Sb}$ & As & $\mathrm{Se}$ & Mo & $\mathrm{Cu}$ & $\mathrm{Pb}$ & $\mathrm{Zn}$ & $\mathrm{Cd}$ & $\mathrm{Bi}$ & Co & $\mathrm{Ni}$ & $\mathrm{Mn}$ \\
\hline & Detection limit & $0.01 \%$ & $0.01 \%$ & $0.01 \%$ & $0.01 \%$ & 0.1 & 0.1 & 0.1 & 0.1 & 0.2 & 0.5 & 0.2 & 0.1 & 0.02 & 0.1 & 0.5 & 1 \\
\hline 102 & MD2-Q1, S & 44 & 0.02 & 28.00 & 65 & 176,000 & 17.5 & 153 & 1.5 & 3.9 & $<0.5$ & 4.2 & 0.7 & 0.09 & $<0.1$ & & 3 \\
\hline \multirow[t]{2}{*}{103} & MD2-Q2. & & 0.05 &, 70 & 25 & 4,000 & 16.8 & 115 & $<0.1$ & 2.8 & $<0.5$ & 4.1 & 0.8 & 0.1 & $<0$ & & 5 \\
\hline & Average $(\mathrm{r}$ & & & 25.85 & & & 17.2 & 134 & 0.8 & 3.4 & $<0.5$ & .2 & & 0.1 & & & 4 \\
\hline 104 & MD2-B & & 5.45 & & & 232 & 73.3 & 9.2 & 9.7 & 96.1 & 40.4 & 321 & & 0.75 & 21.1 & 99. & 873 \\
\hline 05 & D2-B & & 2.86 & & & 47.2 & 20.1 & 1.9 & 1.7 & 54.9 & 35.3 & 77.5 & 0.5 & 0.41 & 24.9 & H1 & 992 \\
\hline 106 & MD2-B & & 9.17 & .32 & 9.6 & 1200 & 18.8 & 4.7 & 24.4 & 118.0 & 7.3 & 33.4 & $<0.1$ & 0.07 & 45 & & 421 \\
\hline 107 & D2- & & 10.6 & 1.37 & 12.9 & 13500 & 4.7 & 4.8 & 2.4 & 123.0 & 11.2 & 54.7 & 0.2 & 0.2 & 59.4 & & 317 \\
\hline 99 & ste wet & & 3.68 & 0.04 & 2.58 & 43.7 & 22.1 & 2.5 & 4.0 & 60.1 & 28.6 & 125 & 0.2 & 0.52 & 9.4 & 35.4 & 299 \\
\hline 101 & MD-BT7, waste, wet & 6.69 & 4.21 & 0.03 & 1.09 & 7.1 & 32.9 & 3.9 & 5.4 & 62.0 & 26.0 & 73.1 & $<0.1$ & 0.6 & 3.6 & 26.8 & 167 \\
\hline \multirow[t]{2}{*}{100} & MD-BT8, waste, wet & & 3.19 & 0.37 & 1.39 & 148 & 46.5 & 6.3 & 6.2 & 45.6 & 21.5 & 80.5 & 0.3 & 0.41 & 4.3 & 37.7 & 303 \\
\hline & Average $(n=7)$ & 7.23 & 5.6 & 0.74 & 5.73 & 2,168 & 31.2 & 4.8 & 7.7 & 80.0 & 24.3 & 109 & 0.5 & 0.42 & 24 & 78.5 & 482 \\
\hline
\end{tabular}

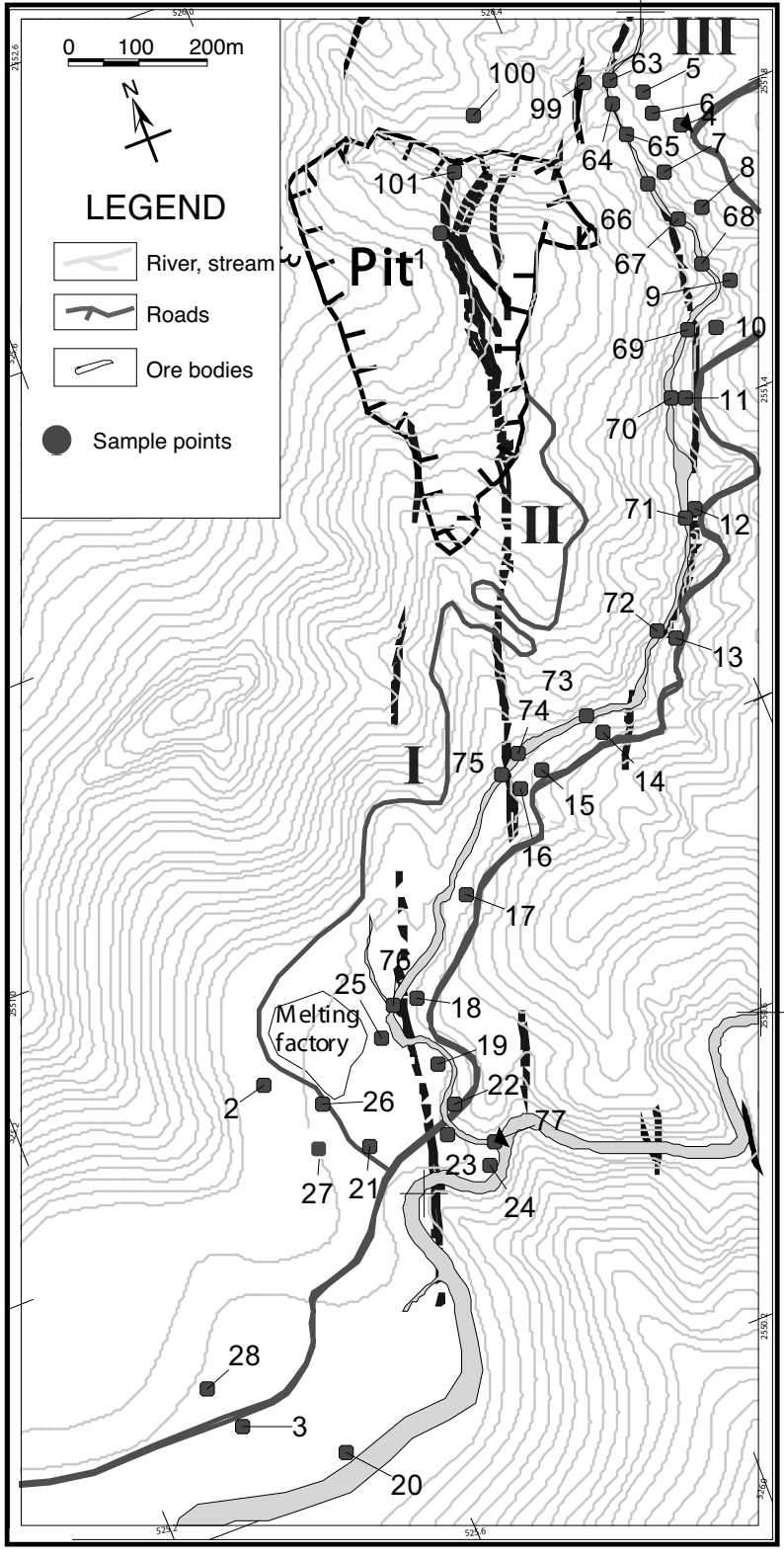

Fig. 2 Locality map of soils and stream sediments in the wet season.

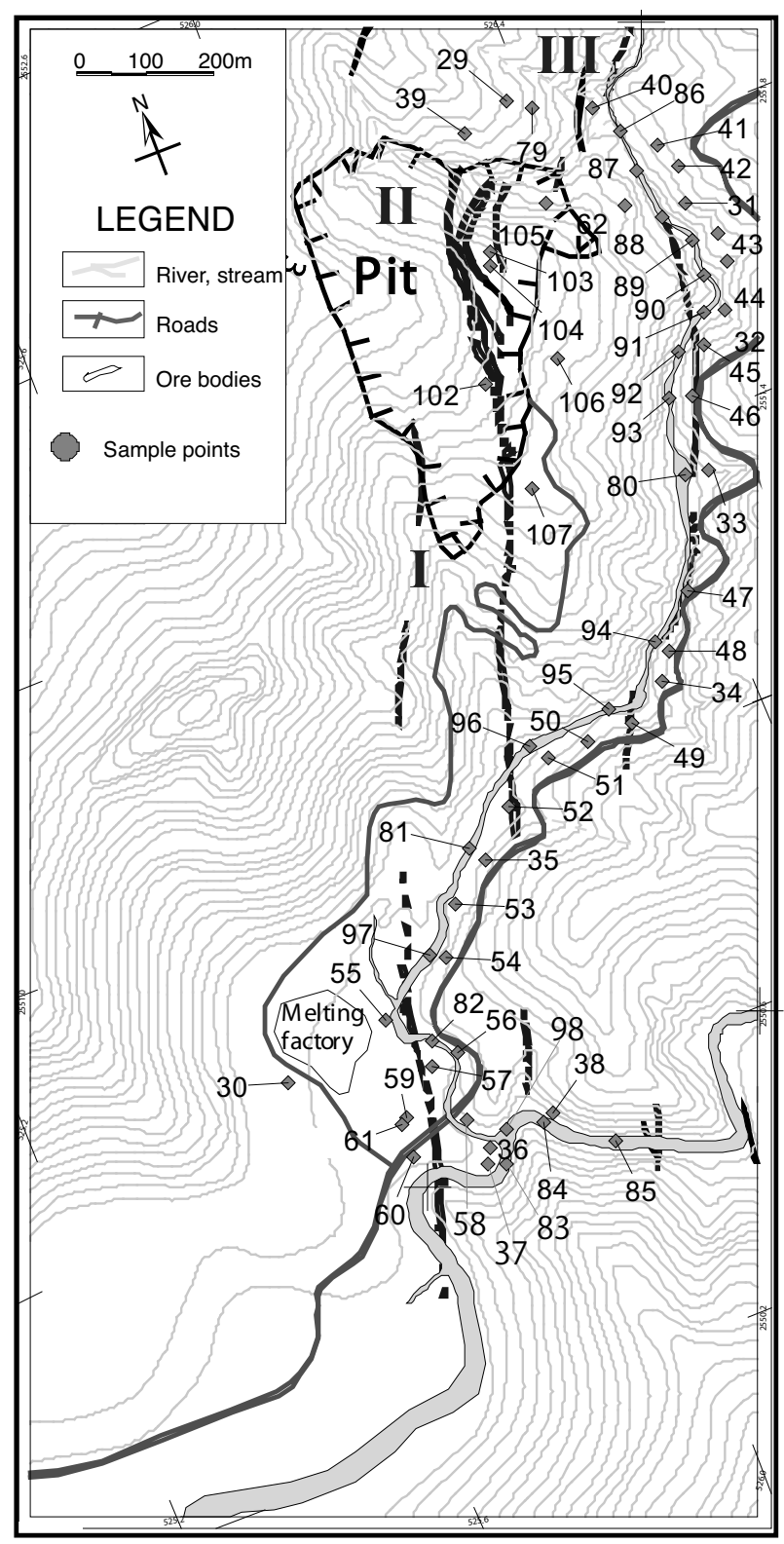

Fig. 3 Locality map of soils and stream sediments in the dry season. 
Table 2 Selected ore components of the soil samples of the wet and dry seasons.

\begin{tabular}{|c|c|c|c|c|c|c|c|c|c|c|c|c|c|c|c|}
\hline \multicolumn{2}{|c|}{ Sample no } & \multirow{2}{*}{$\frac{\mathrm{Ai}}{>10}$} & \multirow{2}{*}{$\begin{array}{r}\mathrm{Fe} \\
3.69\end{array}$} & \multirow{2}{*}{$\begin{array}{r}\mathrm{Sb} \\
2700\end{array}$} & \multirow{2}{*}{$\begin{array}{r}\text { As } \\
31.7\end{array}$} & \multirow{2}{*}{$\frac{\mathrm{Se}}{2.3}$} & \multirow{2}{*}{$\frac{\mathrm{Mo}}{3.8}$} & \multirow{2}{*}{$\begin{array}{r}\mathrm{Cu} \\
42.9\end{array}$} & \multirow{2}{*}{$\begin{array}{r}\mathrm{Pb} \\
34.6\end{array}$} & $\overline{Z n}$ & $\overline{\overline{C d}}$ & $\overline{\mathrm{Bi}}$ & Co & $\overline{\mathrm{Ni}}$ & $\overline{\mathrm{Mn}}$ \\
\hline 1 & MD-D1 wet & & & & & & & & & 63.5 & 0.2 & 0.6 & 3.2 & 24.9 & $\overline{96}$ \\
\hline 2 & MD-D4 & $>10$ & 6.44 & 49 & 61.7 & 2.9 & 4.1 & 71.8 & 94.9 & 224 & 0.5 & 1.0 & 30.8 & 77.5 & 3160 \\
\hline 3 & MD-D5 & 6.66 & 3.37 & 28 & 32.1 & 1.3 & 4.1 & 60.6 & 43.2 & 225 & 0.5 & 0.6 & 15.7 & 49.1 & 2000 \\
\hline 4 & MD-D6 & 8.29 & 3.73 & 19 & 24.3 & 2.6 & 3.4 & 53.4 & 25.4 & 136 & 0.1 & 0.5 & 3.3 & 27.5 & 266 \\
\hline 5 & MD-D7 & 2.91 & 2.60 & 393 & 21.5 & 2.1 & 3.6 & 34.2 & 27.5 & 67 & 0.2 & 0.4 & 4.0 & 21.8 & 188 \\
\hline 6 & MD-D8 & 4.59 & 3.55 & 10 & 17.2 & 1.9 & 3.5 & 48.9 & 27.1 & 84.8 & 0.1 & 0.4 & 12.0 & 30.7 & 488 \\
\hline 7 & MD-D9 & 9.40 & 4.71 & 13 & 17.8 & 2.9 & 5.2 & 75.2 & 35.0 & 123 & 0.2 & 0.6 & 20.9 & 38.1 & 936 \\
\hline 8 & MD-D10 & $>10$ & 4.92 & 100 & 24.1 & 3.6 & 6.3 & 104 & 34.6 & 201 & 0.2 & 0.7 & 26.7 & 55.5 & 2560 \\
\hline 9 & MD-D11 & 8.23 & 4.22 & 7300 & 13.8 & 3.4 & 1.2 & 72.1 & 37.2 & 148 & 0.5 & 0.6 & 20.9 & 44.1 & 1590 \\
\hline 10 & MD-D12 & 7.50 & 3.79 & 1100 & 22.0 & 3.5 & 3.6 & 58.8 & 37.3 & 113 & 0.4 & 0.5 & 28.3 & 44.6 & 1340 \\
\hline 11 & MD-D13 & 7.18 & 3.47 & 5500 & 23.0 & 4.1 & 1.8 & 89.5 & 34.9 & 159 & 1.2 & 0.5 & 26.9 & 70.5 & 1350 \\
\hline 12 & MD-D14 & 0.07 & 4.57 & 90 & 21.0 & 2.9 & 6.0 & 101 & 33.1 & 147 & 0.2 & 0.6 & 23.3 & 48.6 & 2060 \\
\hline 13 & MD-D15 & 8.38 & 4.10 & 7600 & 11.7 & 2.2 & 1.0 & 71 & 33.2 & 105 & 0.3 & 0.5 & 24.2 & 44.7 & 1130 \\
\hline 14 & MD-D16 & 3.15 & 2.52 & 50 & 11.1 & 3.6 & 5.5 & 87.3 & 31.9 & 115 & 0.4 & 0.4 & 26.4 & 54.0 & 1360 \\
\hline 15 & MD-D17 & 6.90 & 4.48 & 261 & 30.5 & 9.0 & 9.1 & 103 & 31.2 & 114 & 0.3 & 0.5 & 33.2 & 44.3 & 1130 \\
\hline 16 & MD-D18 & 7.51 & 3.51 & 41 & 47.3 & 2.3 & 21.0 & 74.7 & 31.2 & 132 & 0.3 & 0.5 & 13.8 & 79.7 & 331 \\
\hline 17 & MD-D19 & $>10$ & 3.74 & 3 & 9.3 & 2.8 & 3.2 & 74.7 & 28.6 & 64.7 & 0.2 & 0.6 & 22.9 & 55.1 & 457 \\
\hline 18 & MD-D20 & 8.12 & 3.97 & 80 & 29.8 & 2.8 & 2.2 & 54.9 & 54.6 & 335 & 3.0 & 0.6 & 45.2 & 106.0 & 2240 \\
\hline 19 & MD-D21 & $>10$ & 4.26 & 45 & 34.7 & 2.0 & 2.5 & 50.9 & 44.5 & 123 & 0.3 & 0.6 & 20.0 & 37.9 & 921 \\
\hline 20 & MD-D22 & 6.54 & 3.03 & 1 & 6.8 & 1.5 & $<0.1$ & 46 & 28.8 & 121 & 0.4 & 0.4 & 15.7 & 43.8 & 677 \\
\hline 21 & MD-D23 & $>10$ & 6.67 & 33 & 49.5 & 1.6 & 2.1 & 76.5 & 69.5 & 211 & 0.4 & 0.9 & 23.5 & 65.6 & 1240 \\
\hline 22 & MD-D24 & $>10$ & 7.28 & 9 & 80.1 & 2.4 & 6.6 & 73.9 & 68.7 & 231 & 0.9 & 1.0 & 30.8 & 103.0 & 3670 \\
\hline 23 & MD-D25 & 7.70 & 4.06 & 6 & 24.6 & 2.1 & 3.2 & 58 & 35.2 & 148 & 0.3 & 0.5 & 22.8 & 56.2 & 908 \\
\hline 24 & MD-D26 & 2.87 & 2.76 & 19 & 10.2 & 1.6 & 2.3 & 41.3 & 23.0 & 105 & 0.4 & 0.3 & 14.1 & 44.3 & 790 \\
\hline 25 & MD-D27 & 6.67 & 3.52 & 26 & 23.5 & 2.0 & 1.7 & 43.6 & 47.3 & 183 & 1.3 & 0.6 & 22.9 & 67.7 & 1200 \\
\hline 26 & MD-D28 & 8.18 & 4.20 & 239 & 28.6 & 1.9 & 1.2 & 50.1 & 73.8 & 175 & 0.5 & 0.7 & 19.9 & 47.0 & 1550 \\
\hline 27 & MD-D29 & $>10$ & 5.55 & 81 & 45.3 & 1.8 & 4.4 & 62.4 & 87.6 & 192 & 0.3 & 0.9 & 27.1 & 60.3 & 2350 \\
\hline 28 & MD-D30 & $>10$ & 5.26 & 46 & 59.9 & 1.5 & 6.7 & 58.5 & 52.1 & 216 & 0.2 & 0.8 & 21.2 & 46.0 & 2060 \\
\hline & Average & $>7.53$ & 4.21 & 923 & 29.0 & 2.7 & 4.3 & 65.7 & 43.1 & 152 & 0.5 & 2.5 & 21.4 & 53.3 & 1359 \\
\hline 29 & MD2-D1, dry & 4.15 & 4.35 & 24 & 32.7 & 3.8 & 7.0 & 68.2 & 26.2 & 86.6 & $<0.1$ & 0.7 & 4.0 & 29.1 & 187 \\
\hline 39 & MD2-D2 & 8.01 & 3.59 & 113 & 47.1 & 3.4 & 7.1 & 52 & 30.2 & 60.5 & 0.1 & 0.8 & 2.2 & 21.8 & 127 \\
\hline 30 & MD2-D3 & $>10$ & 6.02 & 22 & 31.9 & 1.6 & 1.8 & 73.9 & 82.0 & 201 & 0.2 & 0.9 & 27.3 & 61.0 & 2300 \\
\hline 40 & MD2-D4 & 7.35 & 3.17 & 44 & 21.8 & 2.6 & 4.7 & 56.1 & 32.1 & 99.9 & 0.3 & 0.5 & 3.9 & 27.3 & 128 \\
\hline 41 & MD2-D5 & $>10$ & 4.07 & 12 & 25.1 & 3.9 & 7.6 & 53.7 & 38.3 & 86.1 & $<0.1$ & 0.6 & 16.0 & 34.3 & 439 \\
\hline 42 & MD2-D6 & $>10$ & 5.24 & 10 & 23.3 & 3.0 & 6.3 & 76.2 & 34.0 & 109 & 0.3 & 0.6 & 39.1 & 49.4 & 1080 \\
\hline 31 & MD2-D7 & $>10$ & 3.27 & 15 & 16.2 & 2.3 & 6.7 & 46.7 & 49.9 & 113 & 0.2 & 0.6 & 26.2 & 38.1 & 780 \\
\hline 43 & MD2-D8 & 2.46 & 3.58 & 147 & 15.5 & 2.0 & 4.2 & 89.9 & 34.4 & 166 & 0.4 & 0.5 & 29.1 & 44.9 & 2370 \\
\hline 44 & MD2-D9 & 6.84 & 4.49 & 469 & 15.3 & 3.5 & 3.5 & 82 & 37.6 & 125 & 0.2 & 0.6 & 38.7 & 37.7 & 2340 \\
\hline 32 & MD2-D10 & 8.18 & 5.04 & 3300 & 24.3 & 3.3 & 4.3 & 84.2 & 37.2 & 133 & 0.3 & 0.6 & 20.8 & 44.1 & 989 \\
\hline 45 & MD2-D11 & 7.79 & 3.68 & 4700 & 17.5 & 2.8 & 2.8 & 66.6 & 32.1 & 118 & 0.5 & 0.5 & 18.1 & 51.5 & 692 \\
\hline 46 & MD2-D12 & $>10$ & 4.34 & 200 & 35.8 & 1.6 & 4.0 & 36.3 & 56.0 & 139 & 0.5 & 0.7 & 18.4 & 46.9 & 1720 \\
\hline 33 & MD2-D13 & 7.46 & 4.59 & 61 & 23.9 & 2.9 & 1.2 & 67.6 & 36.2 & 190 & 0.5 & 0.6 & 24.9 & 58.3 & 1610 \\
\hline 47 & MD2-D14 & 8.26 & 3.70 & 23 & 18.5 & 3.5 & 6.6 & 70 & 33.8 & 88.4 & 0.3 & 0.6 & 15.1 & 43.2 & 1050 \\
\hline 48 & MD2-D15 & $>10$ & 4.94 & 187 & 18.4 & 4.4 & 9.1 & 136 & 50.2 & 269 & 1.7 & 0.7 & 67.9 & 169.0 & 4010 \\
\hline 34 & MD2-D16 & 8.09 & 3.29 & 85 & 16.4 & 5.8 & 5.1 & 84.4 & 32.6 & 140 & 0.6 & 0.8 & 26.2 & 60.6 & 1650 \\
\hline 49 & MD2-D17 & 9.78 & 5.61 & 19 & 18.7 & 2.2 & 3.9 & 62.6 & 25.6 & 86.7 & $<0.1$ & 0.6 & 4.9 & 36.0 & 185 \\
\hline 50 & MD2-D18 & 5.96 & 4.12 & 15 & 13.6 & 2.0 & 4.3 & 57.9 & 30.7 & 58.6 & $<0.1$ & 0.5 & 5.9 & 37.8 & 161 \\
\hline 51 & MD2-D19 & 4.11 & 2.78 & 33 & 47.8 & 2.1 & 7.2 & 78.1 & 32.3 & 145 & 0.5 & 0.5 & 21.3 & 68.0 & 1680 \\
\hline 52 & MD2-D20 & 6.89 & 3.19 & 89 & 42.8 & 2.1 & 15.1 & 69.5 & 31.2 & 141 & 0.3 & 0.5 & 19.3 & 68.2 & 867 \\
\hline 35 & MD2-D21 & 6.58 & 3.58 & 13 & 48.7 & 2.2 & 16.3 & 93.5 & 32.4 & 162 & 0.5 & 0.6 & 18.2 & 41.8 & 871 \\
\hline 53 & MD2-D22 & 8.19 & 3.77 & 96 & 32.3 & 3.8 & 1.7 & 87.2 & 41.4 & 213 & 2.2 & 0.8 & 24.7 & 76.7 & 1470 \\
\hline 54 & MD2-D23 & 6.36 & 3.06 & 29 & 19.9 & 1.2 & 4.7 & 38.6 & 33.4 & 82.8 & 0.4 & 0.3 & 18.4 & 48.0 & 1820 \\
\hline 55 & MD2-D24 & $>10$ & 3.84 & 65 & 18.4 & 3.9 & 8.2 & 66.7 & 42.5 & 109 & 0.2 & 0.8 & 24.6 & 45.7 & 1580 \\
\hline 56 & MD2-D25 & 8.27 & 4.53 & 42 & 22.9 & 2.2 & 4.2 & 54 & 33.4 & 123 & 0.3 & 0.5 & 27.0 & 43.9 & 1020 \\
\hline 57 & MD2-D26 & 7.78 & 3.59 & 132 & 25.7 & 2.2 & 1.3 & 50.9 & 41.6 & 139 & 0.7 & 0.5 & 17.8 & 48.1 & 1130 \\
\hline 58 & MD2-D27 & 7.72 & 3.77 & 27 & 17.2 & 2.0 & 5.6 & 42.1 & 38.5 & 115 & 0.2 & 0.5 & 11.4 & 30.4 & 452 \\
\hline 36 & MD2-D28 & 6.00 & 2.94 & 17 & 13.3 & 1.7 & 1.1 & 40.1 & 34.6 & 106 & 0.5 & 0.4 & 15.5 & 41.7 & 1290 \\
\hline 37 & MD2-D29 & 2.78 & 3.21 & 27 & 13.6 & 1.3 & 2.8 & 51.1 & 25.7 & 105 & 0.4 & 0.4 & 18.2 & 45.8 & 1560 \\
\hline 38 & MD2-D30 & 6.78 & 3.45 & 35 & 23.3 & 2.1 & 3.2 & 54.8 & 37.6 & 158 & 0.5 & 0.5 & 19.6 & 45.6 & 1340 \\
\hline 61 & MD3-D8 & 6.03 & 20.20 & 1200 & 55.0 & 17.2 & 10.7 & 110 & 25.4 & 1360 & 3.0 & 0.2 & 30.1 & 121.0 & 873 \\
\hline 59 & MD3-D11 & 7.10 & 2.94 & 22 & 14.7 & 1.6 & 1.7 & 45.8 & 69.4 & 177 & 0.8 & 0.6 & 22.9 & 58.3 & 1080 \\
\hline 62 & MD3-D14 & 6.60 & 3.36 & 4 & 12.6 & 1.7 & 1.7 & 47.5 & 75.7 & 133 & 0.5 & 0.5 & 16.1 & 42.3 & 711 \\
\hline & Average & $>7.44$ & 4.40 & 342 & 25.0 & 3.1 & 5.3 & 66.5 & 39.2 & 168 & 0.5 & 0.6 & 21.0 & 52.0 & $\overline{1199}$ \\
\hline
\end{tabular}


Table 3 Selected ore components of the stream sediment samples of the wet and dry seasons.

\begin{tabular}{|c|c|c|c|c|c|c|c|c|c|c|c|c|c|c|}
\hline Sample Nos. & $\mathrm{Al}$ & $\mathrm{Fe}$ & $\mathrm{Sb}$ & As & $\mathrm{Se}$ & Mo & $\mathrm{Cu}$ & $\mathrm{Pb}$ & $\mathrm{Zn}$ & $\mathrm{Cd}$ & $\mathrm{Bi}$ & Co & $\mathrm{Ni}$ & $\mathrm{Mn}$ \\
\hline 63 MD-TT1, wet & 9.27 & 4.52 & 13 & 33.9 & 2.4 & 4.1 & 81 & 28.8 & 146 & 0.2 & 0.5 & 20.9 & 55.3 & 690 \\
\hline 64 MD-TT4 & $>10.0$ & 4.37 & 344 & 69.2 & 7.0 & 4.9 & 133 & 61.3 & 466 & 3.2 & 0.6 & 33.7 & 154.0 & 1740 \\
\hline 65 MD-TT5 & 8.29 & 4.22 & 336 & 77.1 & 6.3 & 5.5 & 102 & 54.6 & 340 & 2.3 & 0.5 & 22.0 & 117.0 & 1330 \\
\hline 66 MD-TT6 & 9.03 & 4.53 & 4000 & 86.2 & 7.6 & 10.7 & 92.7 & 44.2 & 264 & 2.2 & 0.5 & 31.3 & 129.0 & 1860 \\
\hline 67 MD-TT7 & 9.20 & 4.42 & 4200 & 88.0 & 9.9 & 16.6 & 101 & 45.8 & 252 & 2.1 & 0.5 & 19.8 & 108.0 & 1100 \\
\hline 68 MD-TT8 & 6.24 & 3.87 & 3400 & 73.6 & 6.4 & 7.2 & 105 & 47.9 & 322 & 2.3 & 0.5 & 28.2 & 123.0 & 1500 \\
\hline 69 MD-TT9 & 7.68 & 4.22 & 3700 & 76.3 & 7.7 & 11.7 & 100 & 38.4 & 331 & 2.3 & 0.4 & 27.4 & 126.0 & 1420 \\
\hline 70 MD-TT10 & 9.86 & 4.25 & 3700 & 76.0 & 6.5 & 12.8 & 98 & 53.0 & 274 & 2.2 & 0.5 & 27.6 & 120.0 & 1810 \\
\hline 71 MD-TT11 & 8.52 & 4.22 & 4100 & 70.2 & 6.8 & 9.5 & 101 & 43.1 & 282 & 2.3 & 0.5 & 27.7 & 121.0 & 1520 \\
\hline 72 MD-TT12 & 9.20 & 4.22 & 3800 & 68.8 & 6.4 & 7.0 & 105 & 50.1 & 343 & 2.2 & 0.5 & 27.7 & 126.0 & 1640 \\
\hline 73 MD-TT13 & 9.40 & 4.39 & 3300 & 76.3 & 6.2 & 12.4 & 114 & 51.7 & 411 & 2.0 & 0.6 & 30.9 & 129.0 & 1370 \\
\hline 74 MD-TT14 & 9.21 & 4.39 & 3200 & 70.3 & 7.3 & 11.7 & 119 & 56.0 & 460 & 2.7 & 0.6 & 35.0 & 157.0 & 1640 \\
\hline 75 MD-TT15 & $>10.0$ & 4.53 & 3400 & 73.8 & 6.1 & 11.8 & 98.3 & 47.7 & 261 & 1.6 & 0.6 & 25.6 & 107.0 & 1520 \\
\hline 76 MD-TT16 & 9.37 & 4.34 & 215 & 21.6 & 5.4 & 0.3 & 68.6 & 78.4 & 214 & 1.6 & 0.7 & 27.6 & 78.6 & 2360 \\
\hline 77 MD-TT17 & 6.29 & 3.92 & 337 & 60.7 & 6.3 & 5.3 & 91.4 & 35.2 & 324 & 2.9 & 0.5 & 35.9 & 138.0 & 1300 \\
\hline Average & $>8.58$ & 4.29 & 2536 & 68.1 & 6.6 & 8.8 & 100.7 & 49.1 & 313 & 2.1 & 0.5 & 28.1 & 119.0 & 1520 \\
\hline 78 MD2-TT1, dry & 1.90 & 4.62 & 456 & 91.6 & 7.3 & 8.6 & 66.4 & 33.4 & 92.1 & 0.4 & 0.6 & 4.0 & 44.3 & 250 \\
\hline 85 MD2-TT2 & 7.53 & 5.71 & 1800 & 74.3 & 4.2 & 22.4 & 155 & 63.2 & 537 & 2.2 & 0.6 & 38.3 & 153.0 & 1960 \\
\hline 86 MD2-TT3 & 7.34 & 4.25 & 11900 & 90.6 & 10.6 & 7.6 & 95.1 & 48.4 & 221 & 2.4 & 0.7 & 18.8 & 97.7 & 1060 \\
\hline 87 MD2-TT4 & 76 & 6.83 & 2000 & 70.0 & 6.9 & 19.9 & 3.5 & 48.4 & 232 & 1.0 & 0.5 & 18.3 & 93.6 & 1070 \\
\hline 88 MD2-TT5 & 7.86 & 5.13 & 2500 & 71.8 & 5.5 & 19.6 & 91.1 & 54.7 & 219 & 1.4 & 0.5 & 22.2 & 91.9 & 1350 \\
\hline 89 MD2-TT6 & 8.03 & 6.30 & 2600 & 76.8 & 6.3 & 16.8 & 65.4 & 46.4 & 200 & 1.0 & 0.6 & 14.8 & 74.1 & 854 \\
\hline 90 MD2-TT7 & 7.78 & 4.59 & 3500 & 78.4 & 6.7 & 17.3 & 86 & 112.0 & 208 & 1.4 & 0.5 & 26.1 & 102.0 & 1500 \\
\hline 91 MD2-TT8 & 8.41 & 6.44 & 2600 & 72.4 & 6.8 & 16.6 & 78.3 & 44.7 & 240 & 1.3 & 0.5 & 19.8 & 92.5 & 1180 \\
\hline 92 MD2-TT9 & 6.89 & 4.15 & 3300 & 71.1 & 6.0 & 12.7 & 95.4 & 50.3 & 205 & 1.4 & 0.5 & 21.7 & 101.0 & 1220 \\
\hline 79 MD2-TT10 & 9.95 & 4.93 & 2600 & 84.0 & 5.9 & 22.3 & 127 & 76.0 & 287 & 1.4 & 0.7 & 25.3 & 128.0 & 1890 \\
\hline 93 MD2-TT11 & 2.61 & 3.41 & 3300 & 75.6 & 4.9 & 19.5 & 85.4 & 55.8 & 217 & 1.3 & 0.4 & 22.2 & 88.4 & 792 \\
\hline 94 MD2-TT12 & 4.15 & 3.92 & 3500 & 76.1 & 5.8 & 13.0 & 81 & 39.2 & 234 & 1.9 & 0.5 & 28.0 & 104.0 & 1320 \\
\hline 95 MD2-TT13 & 9.80 & 4.99 & 2800 & 81.9 & 5.7 & 25.6 & 112 & 70.1 & 271 & 1.1 & 0.7 & 22.0 & 112.0 & 1800 \\
\hline 90 MD2-TT14 & 8.94 & 9.56 & 2300 & 72.8 & 7.2 & 17.5 & 103 & 48.8 & 536 & 1.5 & 0.6 & 41.7 & 138.0 & 1050 \\
\hline 96 MD2-TT15 & 7.52 & 4.26 & 2600 & 74.7 & 5.7 & 19.8 & 109 & 53.2 & 308 & 1.8 & 0.5 & 33.2 & 125.0 & 1700 \\
\hline 81 MD2-TT16 & 7.28 & 6.23 & 2300 & 64.4 & 5.3 & 10.7 & 93.2 & 47.6 & 738 & 3.2 & 0.5 & 52.7 & 142.0 & 1440 \\
\hline 97 MD2-TT17 & 8.25 & 7.40 & 2200 & 65.3 & 6.6 & 12.7 & 95.2 & 48.7 & 979 & 4.4 & 0.5 & 95.6 & 214.0 & 2020 \\
\hline 82 MD2-TT18 & 4.16 & 2.08 & 16.4 & 13.5 & 0.9 & 0.7 & 22.1 & 23.5 & 64.1 & 0.2 & 0.3 & 9.6 & 23.9 & 682 \\
\hline 83 MD2-TT19 & 6.01 & 2.75 & 135 & 21.3 & 1.7 & 2.7 & 35.5 & 31.1 & 145 & 0.7 & 0.4 & 16.8 & 41.7 & 774 \\
\hline 84 MD2-TT20 & 4.68 & 2.44 & 78.9 & 16.3 & 1.7 & 2.1 & 33.9 & 24.0 & 159 & 0.7 & 0.3 & 19.0 & 47.6 & 622 \\
\hline 98 MD3-B6 (MD3-TT6 & 2.23 & 3.60 & 94.9 & 59.4 & 6.4 & 12.3 & 88.5 & 28.2 & 552 & 4.3 & 0.5 & 24.4 & 161.0 & 921 \\
\hline Average & 6.67 & 4.93 & 2504 & 66.8 & 5.6 & 14.3 & 85.9 & 49.9 & 316 & 1.7 & 0.5 & 27.4 & 103.6 & 1212 \\
\hline
\end{tabular}

to the main antimony mineralization. Another characteristic is very low Al contents and high stibnite components, implying all the feldspars of the original rocks were replaced by stibnite. The mineralized fluids must have been strongly enriched in sulfur and antimony.

\section{Ore components in soil and stream sediments}

Localities of the studied samples are shown in Figs. 2 (wet season) and 3 (dry season). Chemical analyses of the soil and stream sediments were listed in Tables 2 and 3. Stream sediments of Table 3 are listed from the upper stream side to the down stream side. Therefore, the contents increase to the orebody site, then decrease downward.

The stream sediments have smaller variation than the soils in the contents, which is largely due to homogenization in the water relative to the soil samples, as best shown by $\mathrm{Sb}$ contents. The samples were also grouped into those taken in the wet season of May to September and the dry season of October to April. Compared with the average values of the two seasons, antimony seems to be predominant in the wet-season soil samples (932 ppm vs. $342 \mathrm{ppm}$ ). On the other hand, molybdenum is definitely higher in the stream sediments of the dry season (14.3 ppm vs. 8.8 ppm Mo).

Antimony of the soil is sporadically high due to mixing of high-grade ores, but the high values are lowered in the stream sediments (Fig. 4A), which is considered resulted from mechanical mixing of other rocks during sedimentation. Arsenic has a positive correlation with antimony in general, implying both the elements were essentially derived from the mineralized rocks as sulfides. However, the amounts are higher as $67 \mathrm{ppm}$ As (average) in the stream sediments, than the soil of 27 ppm As (average), thus arsenic is considered concentrated as finest grained arsenopyrite in the stream sediments.

Sporadic high values are found in the soils of the wet season and also the stream sediments of the wet season. 

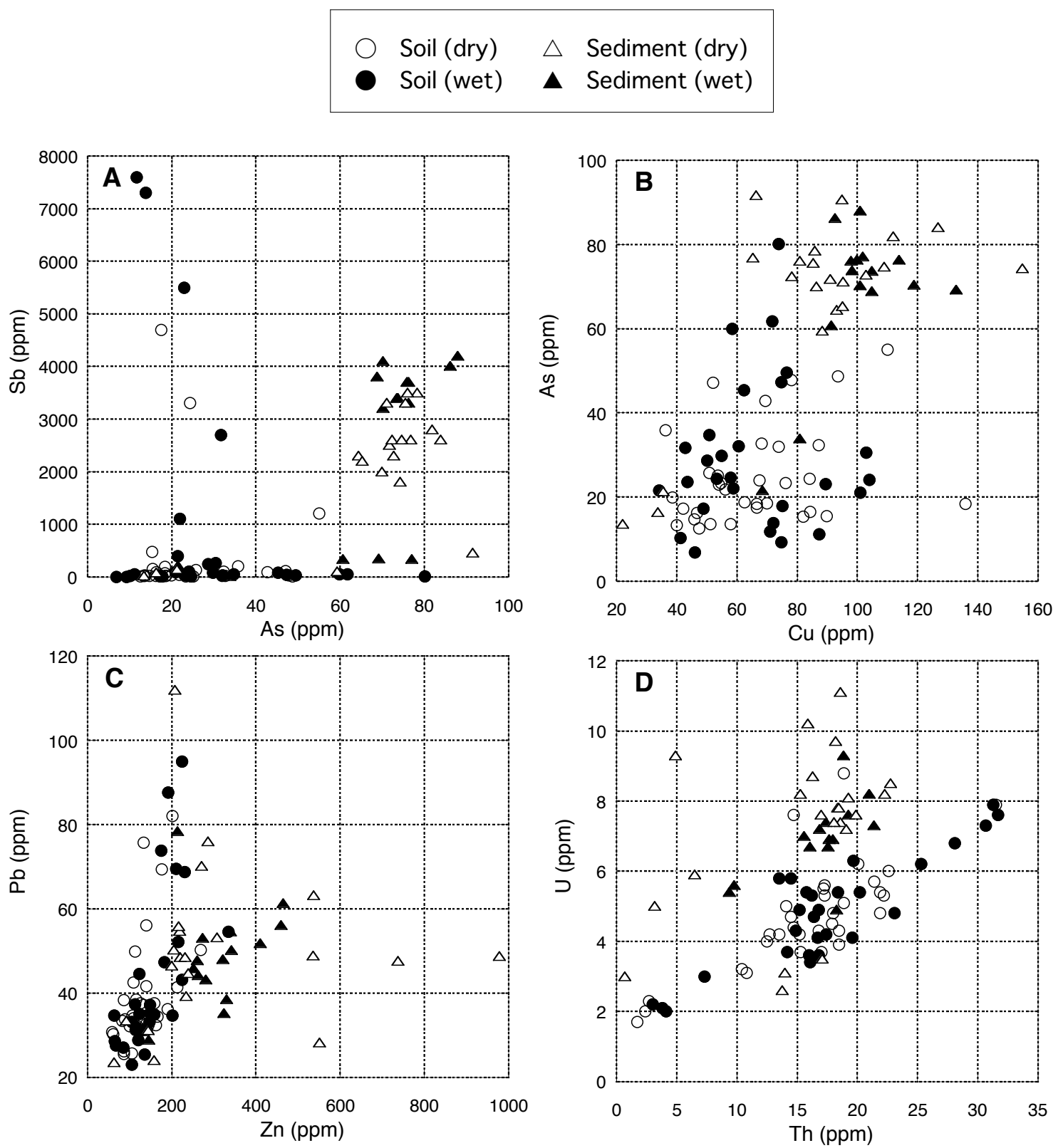

Fig. 4 Variation diagrams of the selected ore components.

A positive correlation of antimony and arsenic of the sediments is unclear on the soil samples (Fig. 4A). Arsenic and copper have broadly positive correlation (Fig. 4B), and stream sediments show higher values in both the elements relative to the soil samples. Lead of the soil samples have a narrow range between 20 and 100 ppm, but zinc contents are scattered especially on those of dry season (Fig. 4C). Thorium and uranium show unique variations. Thorium of wet and dry seasons soils have a good positive variations with uranium. Uranium of sediments is, however, enriched, especially on those of dry season (Fig. 4D), indicating secondary concentration in the element.

\section{Geochemical setting of Sb-mineralized terrain in Japan}

Antimony deposits in Japan are best concentrated in sedimentary terrains of the Outer Zone of Southwest Japan. They are quartz vein or fracture-filling types with simple ore mineralogy of stibnite with quartz. This kind of ore deposits are best concentrated in western Shikoku, where the famous Ichinokawa deposits are located (Ishihara, 2012). The antimony deposits occur in the Sanbagawa metamorphic belt and southward of the Shimanto sedimentary terrains. Antimony contents of these metamorphic and sedimentary rocks were examined by the same analytical method as of the Mae Due samples, and found to have the following range and 
average values of antimony:

Sanbagawa metamorphic rocks ( $\mathrm{n}=3): 0.2 \sim 0.9 \mathrm{ppm}$. Average $0.5 \mathrm{ppm} \mathrm{Sb}$.

Chichibu-Sanbosan, Sandstone (n=4): $<0.1 \sim 2.9 \mathrm{ppm}$. Average $0.9 \mathrm{ppm} \mathrm{Sb}$,

Shale $(\mathrm{n}=6):<0.1 \sim 5.4 \mathrm{ppm}$. Average 1.0 ppm Sb.

Shimanto Belt, North, Sandstone $(\mathrm{n}=2):<0.1 \sim 2.2$ $\mathrm{ppm}$. Average $1.1 \mathrm{ppm} \mathrm{Sb}$,

Shale $(\mathrm{n}=7):<0.1 \sim 5.2 \mathrm{ppm}$. Average $1.2 \mathrm{ppm} \mathrm{Sb}$.

Shimanto Belts, South, Sandstone $(\mathrm{n}=6):<0.1 \sim 1.6$ $\mathrm{ppm}$. Average $0.6 \mathrm{ppm} \mathrm{Sb}$,

Shale $(\mathrm{n}=6):<0.1 \sim 2.6$. Average $0.9 \mathrm{ppm} \mathrm{Sb}$.

The same set of sedimentary rocks is not available in the Mau Due mine area. But the soils of nos. 4, 6, 7 and 8 are located in un-mineralized area at northeastern side of vein III, and they have the range of 10 to 100 $\mathrm{ppm} \mathrm{Sb}$ and average of $36 \mathrm{ppm} \mathrm{Sb}(\mathrm{n}=4)$. Thus, Devonian sediments of north Vietnam, which are supposed to be continental in origin, appear to be one or two order of magnitude higher in trace amounts of antimony than eugeosynclinal sediments of western Shikoku of the Japanese Islands.

\section{Conclusions}

Soils and stream sediments around the Mau Due stibnite deposits were geochemically studied together with some ores and mineralized wastes. The mineralization brought up largely stibnite components. The host sediments seem to have enriched $\mathrm{Sb}$ contents, as compared with eugeosynclinal sediments. Pollution due to As and base metals around the ore deposits is found to be weak.

\section{References}

Ashley, P. M., Craw, D., Graham, B. P. and Chappell D. A. (2003) Environmental mobility of antimony around mesothermal stibnite deposits, New South Wales, Australia and southern New Zealand. Jour. Geochem. Expl., 77, 1-14.

Boyle, R. W. and Jonassan, I. R. (1984) Geochemistry of antimony and its use as an indicator element in geochemical prospecting. Jour. Geochem. Expl., 20, 223-302.

Flynn, H. C., Meharg, A. A., Bowyer, P. K. and Paton, G. I. (2003) Antimony bioavailability in mine soils. Environ. Pollution, 124, 93-100.

Ishihara, S. (2012) On the main antimony ore deposits in Japan and their genetic problems. ShigenChishitsu, 62, 151-161 (in Japanese with English abstract).

Ishihara, S. and Ohno, T. (2011) Present status of antimony mineral resources of this world. ShigenChishitsu, 62, 91-97 (in Japanese with English abstract).

Ishihara, S., Murakami, H. and Li, X. (2011) Indium concentration in zinc ores in plutonic and volcanic environments. Examples at the Dulong and Dachang mines, South China. Bull. Geol. Surv. Japan, 62, 259-272.

Murciego, A. M., Sanchez, A. G., Gonzalez, M. A. R., Gil, E. P., Gordillo, C. T., Fernandez, J. C. and Triguero, T. B. (2007) Antimony distribution and mobility in top soils and plants from polluted Sbmining areas in Extremadura (Spain). Environ. Pollution, 145, 15-21.

Tran Xuyen compiled (2001) Geology and mineral resources of Ma Quan sheet. Scale 1:200,000, Text 42 p., Geol. Surv., Ministry of Industry, Vietnam.

Wang, X., He, M., Xi, J. and Lu, X. (2010) Heavy metal pollution of the world largest antimony mineaffected agricultural soils in Hunan province (China). Jour. Soils Sediments, 10, 827-837.

Received January 11, 2013

Accepted March 22, 2013

\title{
ベトナム北部の Mau Due アンチモン鉱床周辺の重金属元素分布について
}

\author{
石原舜三・Pham Tich XUAN
}

\begin{abstract}
要 旨
ベトナム最北部の Mau Due 鉱床はデボン紀の不純なドロマイト質頁岩中の裂鎼充填性の輝安鉱鉱床である. その地化 学的な研究によると，その鉱化成分は主として $\mathrm{S}$ 及び Sb からなり，若干の As・ベースメタル成分を伴う。土䁃中の $\mathrm{Sb}$ 含有量は変化が激しく，これは鉱石成分の混在によるものと考えられる. 雨季 (5-9 月) と乾季 (10-4 月) に分けられた 採取試料の分析結果, 土壤の $\mathrm{Sb}$ 平均含有量は雨季 $932 \mathrm{ppm}$, 乾季 $342 \mathrm{ppm}$ であって雨季で高く, 一方河川堆積物では, 雨季 2,563 ppm，乾季 $2,504 \mathrm{ppm}$ であり，両者で著しく増加する. As は Sb と同様な挙動をとり，先の含有量は雨季/ 乾季の土壌に扔いて 29/26 ppm と, 絶対量が少ない. 河川堆積物では 68-67 ppm に増加するが, この事実は $\mathrm{Sb}$, As が微 細な硫化物として存在することを示唆している. 日本の地向斜帯の堆積岩と比べてべトナムの堆積岩類は, 微量成分と しての Sbに富んでいる可能性がある.
\end{abstract}

